\title{
Validación del Cuestionario Actitud ante el Conflicto Parental: Autoinformada y Referenciada (ACPar)
}

\author{
Francisca Fariña', Ramón Arce ${ }^{2}$, David Tomé ${ }^{*}$ y Dolores Seijo² \\ 1 Universidad de Vigo \\ 2 Universidad de Santiago de Compostela
}

- Recibido: 23 - 09 - 2019 . Aceptado: 09 - 10 - 2019 . Avance Online: 16 - 10 - 2019

RESUMEN: Antecedentes: En las últimas décadas se han creado diferentes instrumentos que posibilitan valorar el conflicto parental en una pareja o una familia. No obstante, no existe ningún cuestionario que permita evaluar la actitud hacia la resolución de dicho conflicto, pese a su relevancia. Esta variable incide directamente en la gestión del conflicto y en la resolución positiva del mismo; resultando de interés para la práctica de los profesionales que trabajan con familias en conflicto parental. Objetivo: validar empíricamente un cuestionario en español que permita medir la actitud hacia la resolución del conflicto parental. Método: La muestra estuvo formada por 2618 progenitores. Los distintos ítems fueron creados y validados por grupos de expertos. Se realizaron análisis factoriales exploratorios y confirmatorios de forma complementaria. Resultados: Se obtuvo evidencia favorable de un modelo de medida formado por dos factores (8 ítems) que permiten evaluar la actitud propia hacia la resolución de un conflicto parental (autoinformada) y la que se considera que tiene el otro progenitor (referenciada). El ajuste del modelo a los datos fue satisfactorio, del mismo modo que la fiabilidad y validez. Conclusiones: El instrumento resultante es válido y fiable para la medición de la actitud ante el conflicto parental.

PALABRAS CAVE: Cuestionario, Conflicto parental, Actitudes, Propiedades psicométricas, Práctica profesional, Ruptura de pareja, Divorcio.

Validation of the Attitude to Parental Conflict Questionnaire: Self-reported and Referenced (ACPar)

ABSTRACT: Background: Recently, several scales have been sesigned to evaluate the parental conflict in a couple or in a family. Nevertheless and despite its relevance, there is no measure instrument to evaluate the attitude towards the conflict resolution. This variable has a direct effect in the conflict management and its positive resolution, being of interest for practitioners working with families in parental conflict. Objective: The empirical validation of a questionnaire that allows to measure, in Spanish, the attitude towards the resolution of the parental conflict. Method: The sample consisted of 2618 parents. The different items were created and validated by groups of experts. Exploratory and confirmatory factorial analyses were carried out in a complementary manner. Results: Favourable evidence was obtained of a measurement model comprised by two factors ( 8 items) that allow the evaluation of one's own attitude towards the resolution of a parental conflict (self-reported) and the considered to have the other parent (referenced). The adjustment of the model to data was satisfactory, in the same way as the validity and reliability. Conclusions: The resulting questionnaire is valid and reliable for the measurement of the attitude towards the parental conflict.

KEY WORDS: Questionnaire; Parental conflict; Attitudes; Psychometric properties; Professional practice, Couple breakdown, Divorce.

La calidad de la relación de pareja es ampliamente reconocida como la piedra angular del funcionamiento familiar adaptativo

*Correspondence: David Tomé.

Universidad de Vigo.

CP: 36002, Pontevedra, España

E-mail:davtome@pontevedra.uned.es

(C) 2020 Sociedad Universitaria de Investigación en Psicología y Salud. Publicado por Consejo General de Colegios Oficiales de Psicólogos, España. Este es un artículo Open Access
bajo la CC BY-NC-ND licencia (http://creativecommons.org/licencias/by-nc-nd/4.0/).
(Gao, Du, Davies, y Cummings, 2018). Por el contrario, el conflicto interparental, entendido como cualquier diferencia, desacuerdo, o discusión sobre un tema de la vida familiar, incluyendo todo tipo de conflictos psicológicos y físicos (Cummings y Davies, 2002; Khurshid, Peng, y Wang, 2019; Martínez-Pampliega, Ugarte, Merino, y Herrero-Fernández, 2019), 
puede facilitar la presencia de emociones hostiles entre los miembros de la pareja. Estas, a su vez, pueden acompañarse de agresiones físicas, verbales o psicológicas, así como de estrategias deficientes para la reducción del conflicto (Tavassolie, Dudding, Madigan, y Thorvardarson, 2016; Tu, Erath, y El-Sheikh, 2016).

Todas las parejas estables con descendencia, con independencia de que exista vínculo matrimonial o no, experimentan, a lo largo de su relación, diferentes conflictos (Dillon et al., 2015); así, los niños a menudo se encuentran expuestos a ellos, pudiendo perjudicar su crianza, al comportamiento parental y a la relación con sus progenitores (Shamir, Schudlich, y Cummings, 2001). De esta manera, se considera el conflicto interparental como un importante factor adverso para los hijos (Cummings y Davies, 2010). Sin embargo, cuando las parejas comparten amabilidad, confianza y comprensión, los conflictos entre ellos se resuelven (Dillon et al., 2015) y pueden gestionarlos de forma positiva, amigable y constructiva lo que, a su vez, incide en la crianza de los hijos, llegando a aumentar su sentimiento de seguridad y bienestar en la familia (Zemp, Bodenmann, y Cummings, 2016). En este sentido, los conflictos pueden promover el crecimiento personal, incluso en familias separadas (Treloar, 2019). No obstante, esto no sucede cuando los progenitores tienen una actitud hostil y utilizan tácticas destructivas, como agresión verbal o física, o expresión de la ira, en cuyo caso se producen reacciones emocionales y de conducta que repercuten negativamente en los descendientes menores de edad (Camisasca, Miragoli, y Di Blasio, 2016; Gao et al., 2019; Zhou y Buehler, 2017). La existencia de conflicto en el hogar es un factor de riesgo para niños y adolescentes, especialmente cuando ocurre de manera frecuente, intensa y no se resuelve adecuadamente (Cutrín, Maneiro, Sobral, y Gómez-Fraguela, 2019; Gallego, Novo, Fariña, y Arce, 2019). Facilita la inadaptación psicológica (Camisasca, Miragoli, y Di Blasio, 2019), asociándose a largo plazo con problemas externalizantes, tal como agresividad, vandalismo, conductas desadaptadas, bajo rendimiento académico; e internalizantes, como síntomatología ansioso- depresiva, baja autoestima, o malestar psicológico (Gómez-Ortiz, Romera, JiménezCastillejo, Ortega-Ruiz, y García-López, 2019; Lucas-Tompson, Lunkenheimer, y Dumitrache, 2017; Yap y Jorm, 2015). Esto puede deberse a que los subsistemas marital y paterno-filial son predictores interdependientes de la gestión emocional de los menores (Frankel, Umemura, Jacobvitz, y Hazen, 2015). Los progenitores que presentan alto grado de conflicto entre ellos, dejan de colaborar en las tareas de crianza, su práctica parental es inconsistente e ineficiente, pudiendo suponer una mayor exposición de los hijos al conflicto (Lamela, Figueiredo, Bastos, y Feinberg, 2016; Tavassolie et al., 2016).

En procesos de ruptura de pareja el alto conflicto parental se ha considerado uno de los principales factores de riesgo para la inadaptación de los descendientes, relacionándose con problemas de comportamiento social y escolar (Corrás et al., 2017; Van der Wal, Finkenaver, y Visser, 2019), así como con el sufrimiento de problemas de salud física y psicológica (Martinón et al., 2017; Seijo, Fariña, Corras, Novo, y Arce, 2016).

En suma, como señalan Zemp et al. (2016), el conflicto interparental destructivo, atendiendo a los resultados de la investigación, es una forma de estrés que afecta a los hijos en todas las etapas del desarrollo; siendo probablemente el factor de riesgo familiar de mayor prevalencia, incluso con mayor impacto que el divorcio. De esta manera, la gestión del conflicto interparental se convierte en una prioridad para los profesionales que trabajan desde diferentes ámbitos con estas familias; como puede ser en la evaluación de custodia (Nielsen, 2017), la coordinación de parentalidad (Quigley y Cyr, 2017), la mediación familiar (Kiøs y Weie-Oddli, 2018), la terapia infantil (Zemp et al., 2016), o la terapia de familia (Smyth y Moloney, 2019; Tavassolie et al., 2016).

El conflicto, en el contexto internacional, se valora a través de instrumentos específicos bien para ser cumplimentados por progenitores como las Conflicts and Problem-Solving Scales (Kerig, 1996), o por los hijos e hijas como la Children's Perception of Interparental Conflict Scale (CPIC; Grych, Seid, y Fincham, 1992) o el Interpersonal Conflict Questionnaire (Laursen, 1993). No 
obstante, el conflicto entre ambos progenitores también puede ser medido mediante factores incluidos en otros cuestionarios que cuantifican variables de pareja o familiares, como el Locke's Marital Adjustment Test (Kimmel y Van Der Veen, 1974) o el Revised Conflict Tactics Scale (Straus, Lamby, Boney-McCoy, y Savage, 1995). Para los hispanohablantes también existen instrumentos que cuentan con factores para evaluar el conflicto familiar y parental, entre ellos, las Escalas de Clima Social y Familiar (Moos, Moos, y Trickett, 1984), igualmente cuestionarios que evalúan la relación con la expareja como el Cuestionario de Adaptación al Divorcio-Separación (Yárnoz-Yaben y CominoGonzález, 2010). También puede ser medido cuantificando el número de conflictos existentes informados por los progenitores (Parra-Jiménez y Oliva-Delgado, 2002; Motrico, Fuentes, y Bersabé, 2001), o con la participación de los hijos e hijas, aplicando la adaptación del CPIC (Iraurgi et al., 2008). Sin embargo, aunque para trabajar el conflicto interparental los profesionales necesitan conocer la actitud de cada progenitor hacia la resolución del mismo y la percepción que tienen sobre la actitud del otro, no disponen en español de instrumentos de medida válidos y fiables. Por este motivo, diseñamos un estudio dirigido a la creción y validación de un instrumento psicométrico que permita evaluar la actitud de los progenitores hacia la resolución de un conflicto parental, pudiendo analizar tanto la que mantiene cada uno (autoinformada) como la que considera que tiene el otro (referenciada). El cuestionario resultante permitirá a terapeutas familiares, psicólogos forenses de familia, mediadores familiares, coordinadores de parentalidad o educadores familiares, obtener una evaluación fiable y válida de las actitudes hacia la resolución del conflicto de los progenitores en un conflicto parental, conocimiento que resulta fundamental para la gestión y resolución positiva del mismo.

\section{MÉTODO}

\section{-PARTICIPANTES}

La muestra estuvo compuesta por 2618 padres y madres españoles. En la Tabla 1 se ofrecen los datos acerca de sus características sociodemográficas.

\begin{tabular}{|c|c|c|}
\hline \multicolumn{3}{|c|}{$\begin{array}{l}\text { Tabla } 1 \\
\text { Características sociodemográficas de los participantes }\end{array}$} \\
\hline Variable & & $N(\%)$ \\
\hline Edad del hijo/a & \multicolumn{2}{|c|}{$M=7.13(D T=2.73)$. Rango $1-12$} \\
\hline \multirow{2}{*}{ Sexo del hijo/a } & Varón & $1311(50.1 \%)$ \\
\hline & Mujer & $1289(49.2 \%)$ \\
\hline \multirow{4}{*}{ Relación con el hijo/a } & Padre biológico o padre adoptivo & $459(17.5 \%)$ \\
\hline & Madre biológica o madre adoptiva & $2127(81.2 \%)$ \\
\hline & Padrastro & $2(.1 \%)$ \\
\hline & Madrastra & $19(.7 \%)$ \\
\hline \multirow{6}{*}{ Estado civil } & Casado/a & $1934(73.9 \%)$ \\
\hline & Conviviendo en pareja & $353(13.5 \%)$ \\
\hline & Divorciado/a & $222(8.5 \%)$ \\
\hline & Soltero/a & $84(3.2 \%)$ \\
\hline & Viudo/a & $9(.3 \%)$ \\
\hline & Otro & $9(.3 \%)$ \\
\hline \multirow{4}{*}{ Estado familiar } & Ambos progenitores biológicos o adoptivos & $2257(86.2 \%)$ \\
\hline & $\begin{array}{l}\text { Un progenitor biológico o adoptivo y un padrastro o } \\
\text { madrastra }\end{array}$ & $91(3.5 \%)$ \\
\hline & Familia de progenitores solteros & $103(3.9 \%)$ \\
\hline & Otro & $129(4.9 \%)$ \\
\hline \multirow{2}{*}{ Hijos/as de relaciones previas } & Sí & $112(4.3 \%)$ \\
\hline & No & $2294(87.6 \%)$ \\
\hline
\end{tabular}




\section{- INSTRUMENTOS DE MEDIDA}

Los datos sociodemográficos presentados en la Tabla 1 se recogieron a través de un cuestionario creado ad hoc. mientras que el instrumento Actitud ante el Conflicto Parental, Autoinformada y Referenciada (ACPar) se diseñó siguiendo las distintas fases de creación de test propuestas por Muñiz y Fonseca-Pedrero (2019) y los estándares para pruebas educativas y psicológicas (American Educational Research Association, American Psychological Association, \& National Council on Measurement in Education, 2014).

En primer lugar, se determinó la finalidad del test, sabiendo que la variable a observar era la percepción de la actitud propia y ajena de los progenitores ante el conflicto entre ellos. A continuación, se especificaron las características que debían tener los ítems del test y su formato como ítems de clasificación tipo Likert, con 11 alternativas de respuesta, donde 0.- Nada o ninguno; y 10.- Máximo o total. A la hora de redactar los ítems, un grupo de 3 expertos en los ámbitos de conflicto, mediación familiar, parentalidad y coparentalidad positiva, evaluación forense y coordinación de parentalidad, crearon 17 ítems (ver la relación en el Anexo 1) que no fueran ambiguos ni sesgados, buscando expresar una única idea por enunciado, y sin dobles negaciones.

Una vez redactados los ítems, otro grupo de 21 expertos $168.2 \%$ mujeres y $27.3 \%$ hombres) analizó los aspectos de calidad de los ítems, juzgando su idoneidad y su inclusión definitiva en el cuestionario, en una escala de 1 (muy en desacuerdo) a 5, (muy de acuerdo) con la inclusión del ítem. Los criterios de inclusión para formar parte de esté comité fueron: 1) No haber participado en la elaboración inicial de los ítems; 2) Haber trabajado 5 años en ámbitos de familia y parentalidad; 3) Tener un título de grado o licenciatura en áreas de conocimiento relacionadas con la familia o la gestión de conflicto. El grado medio de acuerdo interiveces para los 17 ítems fue de 4.71 (DT = $0.12)$, con una correlación intra-clase de .889 y un porcentaje de acuerdo del $94.11 \%$.

Una vez revisados los ítems, se redactó la versión preliminar, y se realizaron entrevistas con un grupo piloto de padres y madres (10 madres y 10 padres) a quienes se les administró el cuestionario de forma individual mediante lápiz y papel. Los participantes del estudio piloto no mostraron dificultad alguna en cumplimentar el cuestionario, ni en lo relativo a la comprensión de los ítems. Finalmente, el cuestionario se administró a la muestra definitiva de participantes.

\section{-PROCEDIMIENTO}

Previamente al inicio de la investigación se obtuvo la aprobación por parte del Comité de Bioética de la Universidad de Santiago de Compostela. Posteriormente se contactó con una selección de centros de educación primaria de carácter público, privado y concertado de diferentes entornos socioeconómicos y localizaciones geográficas. La dirección de los centros informó a las familias acerca de los objetivos de la investigación, así como del carácter anónimo y voluntario de la recogida de datos.

Las instrucciones para cumplimentar el cuestionario fueron las mismas para todos los padres y madres, y estaban escritas en el documento donde debían responder. A aquellos que manifestaron su intención de participar se les envió el cuestionario a cumplimentar y un documento de consentimiento informado, en el que se autorizaba el uso de los datos aportados para los objetivos de la investigación.

\section{-ANÁLISIS DE DATOS}

Los análisis de datos se llevaron a cabo de forma secuencial a través del paquete estadístico IBP SPSS versión 25 y IBM AMOS versión 25. En primer lugar, se calcularon los estadísticos descriptivos de los 17 ítems iniciales. A continuación, se implementó un análisis factorial exploratorio, que dio lugar a la selección de 8 ítems para la versión definitiva. Después, se realizaron procesos de análisis factorial confirmatorio para obtener criterios adicionales del ajuste del cuestionario. La validez convergente y discriminante se calculó a través de la varianza media extraída. Finalmente, se obtuvo la fiabilidad de los 
factores del modelo de medida a través de dos perspectivas: consistencia interna y fiabilidad compuesta. En cuanto al tamaño de la muestra, la mínima recomendada para ejecutar un análisis factorial conformatorio es de 200 casos para modelo teóricos y 300 casos para modelo poblacionales (Kline, 2011 ; Myers, Ahn, y Jin, 2011). Basándonos en estas directrices, la muestra de 2618 participantes en este estudio es adecuada para comprobar las propiedades psicométricas del cuestionario. Asimismo, la razón entre el número de participantes (2618) y el total de parámetros a estimar del modelo (47.6) supera el criterio, $>5$, de Kline (2011).

\section{RESULTADOS}

\section{•ESTADÍSTICOS DESCRIPTIVOS}

En la Tabla 2 se recogen los valores mínimos y máximos obtenidos en los 17 ítems iniciales del cuestionario, así como los estadísticos de la media (M) y desviación típica (DT). Los valores de las medias estuvieron en un rango de 5.49 (ítem 2) a 9.25 (ítem 17), mientras que las desviaciones típicas tuvieron valores iguales o inferiores a 4.11.

\section{-ANÁLISIS FACTORIAL EXPLORATORIO}

El valor de la medida de adecuación muestral, $\mathrm{KMO}=0.833$, la Prueba de esfericidad de Bartlett, $\chi^{2}(136)=33798.41$, $p<.001$ y el determinante de la matriz de correlaciones, .000, informaron de la viabilidad de someter los datos a una reducción en dimensiones, análisis factorial (Tabanick y Fidell, 1989), que se llevó a cabo con el método de extracción de "componentes principales" y el método de rotación apliacdo fue "varimax con normalización de Kaiser". Como criterio de selección de las cargas factoriales se tomó .40 (Pituch y Stevens, 2016). En la Tabla 3 se presenta la rotación que convergió en 6 iteraciones, mientras que en la Tabla 4 aparecen las puntuaciones de la diagonal de la matriz de las correlaciones anti-imagen.

Con el objetivo de determinar el número de factores, se tuvieron en cuenta los siguientes criterios de inclusión: 1) los autovalores son más grades que la unidad; 2) los factores tienen un mínimo de tres ítems; 3) Una interpretación sustantiva basada en el contenido de los ítems agrupados.

Tabla 2

Estadísticos descriptivos de los ítems

\begin{tabular}{|c|c|c|c|c|}
\hline Í́tems & Mínimo & Máximo & M & DT \\
\hline ÍTEM 1 & .0 & 10 & 5.69 & 4.11 \\
\hline ÍTEM 2 & .0 & 10 & 5.49 & 4.11 \\
\hline ÍTEM 3 & .0 & 10 & 8.63 & 2.49 \\
\hline ÍTEM 4 & .0 & 10 & 9.02 & 2.00 \\
\hline ÍTEM 5 & .0 & 10 & 8.77 & 2.01 \\
\hline ÍTEM 6 & .0 & 10 & 8.87 & 1.92 \\
\hline ÍTEM 7 & .0 & 10 & 8.32 & 2.40 \\
\hline ÍTEM 8 & .0 & 10 & 8.32 & 2.40 \\
\hline ÍTEM 9 & .0 & 10 & 8.23 & 2.42 \\
\hline ÍTEM 10 & .0 & 10 & 8.19 & 2.43 \\
\hline ÍTEM 1 1 & .0 & 10 & 5.94 & 2.41 \\
\hline ÍTEM 12 & .0 & 10 & 5.99 & 2.33 \\
\hline ÍTEM 13 & .0 & 10 & 6.47 & 3.41 \\
\hline ÍTEM 14 & .0 & 10 & 5.69 & 2.38 \\
\hline ÍTEM 15 & .0 & 10 & 5.50 & 2.32 \\
\hline ÍTEM 16 & .0 & 10 & 7.31 & 3.35 \\
\hline ÍTEM 17 & .0 & 10 & 9.25 & 1.87 \\
\hline
\end{tabular}


Tabla 3

Matriz factorial rotada

\begin{tabular}{|c|c|c|c|c|c|c|}
\hline \multirow[t]{2}{*}{ Items } & \multicolumn{6}{|c|}{ Factor } \\
\hline & 1 & 2 & 3 & 4 & 5 & 6 \\
\hline ÍTEM 9 & .914 & & & & & \\
\hline ÍTEM 8 & .919 & & & & & \\
\hline ÍTEM 10 & .907 & & & & & \\
\hline ÍTEM 7 & .848 & & & & & \\
\hline ÍTEM 5 & & .835 & & & & \\
\hline ÍTEM 4 & & .823 & & & & \\
\hline ÍTEM 6 & & .791 & & & & \\
\hline ÍTEM 3 & & .717 & & & & \\
\hline ÍTEM 17 & & .463 & & & & \\
\hline ÍTEM 1 & & & .880 & & & \\
\hline ÍTEM 2 & & & .853 & & & \\
\hline ÍTEM 11 & & & & .819 & & \\
\hline ÍTEM 12 & & & & .807 & & \\
\hline ÍTEM 13 & & & & & .765 & \\
\hline ÍTEM 16 & & & & & .758 & \\
\hline ÍTEM 15 & & & & & & .744 \\
\hline ÍTEM 14 & & & & & & .674 \\
\hline Autovalores & 6.126 & 1.998 & 1.843 & 1.481 & 1.350 & 1.077 \\
\hline
\end{tabular}

Tabla 4

Diagonal de la Matriz de Correlaciones Anti-Imagen

\begin{tabular}{|c|c|}
\hline Item & Correlación anti-imagen \\
\hline Ítem 1 & .508 \\
\hline Ítem 2 & .507 \\
\hline Ítem 3 & .885 \\
\hline Ítem 4 & .803 \\
\hline Ítem 5 & .856 \\
\hline Ítem 6 & .866 \\
\hline Ítem 7 & .929 \\
\hline Ítem 8 & .900 \\
\hline Ítem 9 & .885 \\
\hline Ítem 10 & .885 \\
\hline Ítem 11 & .702 \\
\hline Ítem 12 & .678 \\
\hline Ítem 13 & .548 \\
\hline Ítem 14 & .724 \\
\hline Ítem 15 & .740 \\
\hline Ítem 16 & .525 \\
\hline Ítem 17 & .968 \\
\hline
\end{tabular}


Por lo tanto, el modelo quedaría configurado por dos factores que explican el $47.79 \%$ de la varianza. De acuerdo con la interpretación sustantiva, el primer factor se llamará "Actitud Autoinformada (AA)" incluyendo a los ítems 3, 4, 5, y $6(M=8.82$, $D T=1.85)$. El ítem 17 se elimina del factor debido a que presenta una carga factorial baja en relación a los demás y su contenido no se corresponde con el del resto de ítems, lo que corrobora la baja carga factorial. Por otro lado, el segundo factor se llamará "Actitud Referenciada $(A R)$ " incluyendo los ítems 7, 8, 9 y 10 ( $M=8.26$, DT = 2.33). En la Tabla 5 se ofrecen los valores de las correlaciones ítemtotal. En los subsiguientes análisis, los ítems que no formaron parte de estos dos factores fueron desestimados.

\section{-ANÁLISIS FACTORIAL CONFIRMATORIO}

Los análisis factoriales exploratorios y confirmatorios se usaron de forma complementaria, siguiendo lo propuesto por Gorsuch (1997). Por lo tanto, en función de las relaciones entre ítems y factores del análisis exploratorio, se especificó el modelo de la Figura 1.

El modelo se sobre-identificó con 36 elementos en la matriz de varianzas-covarianzas con 19 parámetros para la estimación (8 cargas factoriales, 8 varianzas de error y una correlación entre factores), con 17 grados de libertad.

Con el objetivo de evitar la infraidentificación del modelo, se fijaron las varianzas de los factores y errores a la unidad, dejando libres 8 cargas factoriales y 8 correlaciones.

Tabla 5

Correlaciones ítem-total corregidas

\begin{tabular}{|c|c|c|}
\hline Factor & Item & Correlación ítem-total corregida \\
\hline \multirow{3}{*}{ 1. Actitud Autoinformada } & Ítem 3 & .699 \\
\cline { 2 - 3 } & Ítem 4 & .822 \\
\cline { 2 - 3 } & Ítem 5 & .828 \\
\cline { 2 - 3 } & Ítem 6 & .911 \\
\hline \multirow{3}{*}{ 2. Actitud Referenciada } & Ítem 7 & .954 \\
\cline { 2 - 3 } & Ítem 8 & .951 \\
\cline { 2 - 3 } & Ítem 9 & .944 \\
\cline { 2 - 3 } & Ítem 10 & \\
\hline
\end{tabular}

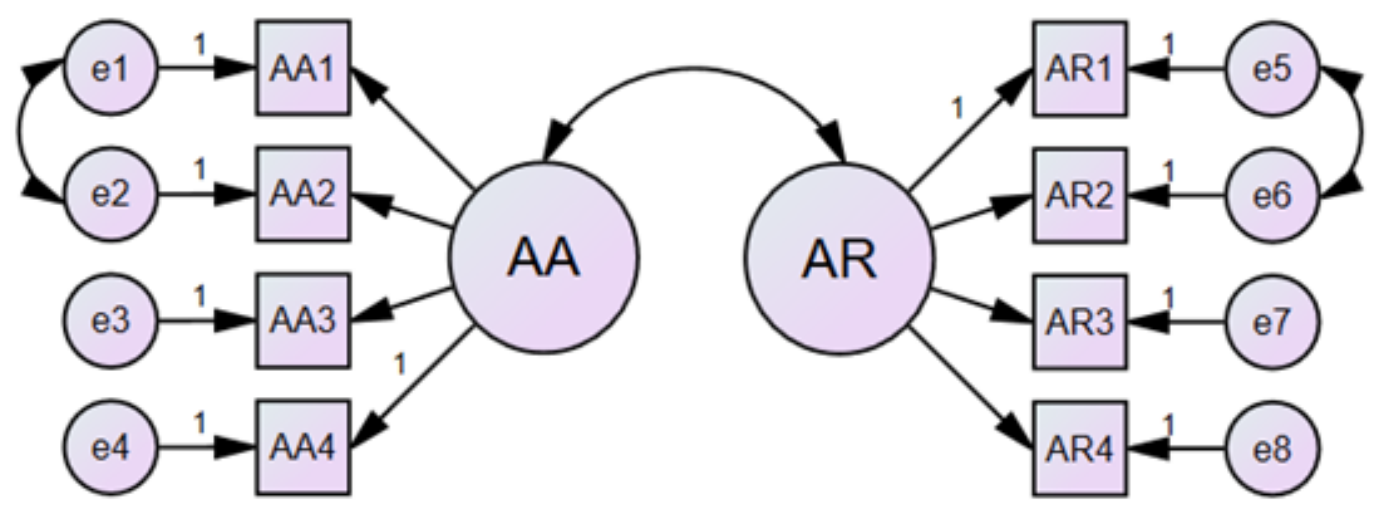

Figura 1: Modelo factorial 
Para conseguir un mejor ajuste del modelo a los datos, se re-especificó el modelo original añadiendo correlaciones entre los errores de los ítems 1 y 2 del factor Actitud Autoinformada $(r=.48)$, y los errores de los ítems 1 y 2 del factor Actitud Referenciada ( $r=.41$ ).

Los parámetros se estimaron por el Método de Máxima Verosimilitud. La Tabla 6 ofrece los valores de las cargas factoriales $(\lambda)$ y de las varianzas de error $(\delta)$. Se obtuvo significación estadística para todos los parámetros ( $p<$ $.001)$. Las correlaciones ítem-total corregidas fueron superiores a .50 para todos los ítems, excepto el ítem 3 (.41). Se estableció una correlación positiva y significativa entre los dos factores del cuestionario $(r=.62 ; p<.001)$.

Los índices de bondad de ajuste para el modelo fueron satisfactorios: $\chi^{2}=212.958(p$ $<.001) ; \chi^{2} / g l=12.527$; Goodness of Fit Index $(\mathrm{GFI})=.981$; Tucker-Lewis Index $(\mathrm{TLI})=.987$; Comparative Fit Index (CFI) = .992; Normed Fit Index $(\mathrm{NFI})=.992$; Root Mean Square Error of Approximation (RMSEA) $=.066$ [IC 90\% .059, .074]; Standardized Root Mean Square Residual $(\mathrm{SRMR})=.0261$.

\section{-VALIDEZ CONVERGENTE Y DISCRIMINANTE}

Para evaluar la validez convergente de cada uno de los factores, se calculó la varianza media extraída (VME). La VME del Factor Actitud Autoinformada fue de .694, mientras que la VME del factor Actitud Referenciada fue de .904 . En este caso, los indicadores son satisfactorios al ser mayores que .5 (Fornell y Larcker, 1981)

En cuanto a la validez discriminante, la VME de ambos factores es superior al cuadrado de la correlación entre ellos (.415), por lo tanto también constituye un buen indicador (Fornell y Larcker, 1981)

\section{-ANÁLISIS DE LA FIABILIDAD}

El cálculo de la fiabilidad para cada uno de los factores se realizó desde dos perspectivas: el coeficiente de alpha de Cronbach y la fiabilidad compuesta. En la Tabla 7 se presentan los resultados, los cuales fueron satisfactorios en todos los casos al superar el umbral de 7 (Carmines y Zeller, 1979; Nunnally, 1978).

Tabla 6

Cargas factoriales y varianzas de error

\begin{tabular}{|c|c|c|c|}
\hline \multirow{2}{*}{ Factor } & Item & Carga factorial $(\lambda)$ & Varianza de error $(\delta)$ \\
\hline \multirow{3}{*}{ 1. Actitud Autoinformada } & AA1 & .644 & .585 \\
\cline { 2 - 4 } & AA2 & .781 & .390 \\
\cline { 2 - 4 } & AA3 & .941 & .115 \\
\cline { 2 - 4 } & AA4 & .930 & .135 \\
\hline \multirow{3}{*}{ 2. Actitud Referenciada } & AR1 & .905 & .181 \\
\cline { 2 - 4 } & AR 2 & .951 & .096 \\
\cline { 2 - 4 } & AR 3 & .976 & .047 \\
\hline
\end{tabular}

Tabla 7

Análisis de fiabilidad

\begin{tabular}{|c|c|c|}
\hline Factor & Alpha de Cronbach & Fiabilidad compuesta \\
\hline 1. Actitud Autoinformada & .900 & .899 \\
\hline 2. Actitud Referenciada & .976 & .974 \\
\hline Total & .932 & .969 \\
\hline
\end{tabular}




\section{DISCUSIÓN}

Este estudio presenta el cuestionario Actitud ante el Conflicto Parental, Autoinformada y Referenciada (ACPar), de utilidad para la práctica profesional, al permitir conocer la actitud de cada progenitor hacia la resolución del conflicto interparental (autoinformada) y la percepción que tienen sobre la actitud del otro (referenciada). Todos los indicadores de bondad de ajuste del modelo han sido satisfactorios (Schermelleh-Engel, Moosbrugger, y Müller, 2003). De igual manera sucede con la validez convergente del cuestionario, que permite concluir que el conjunto de indicadores mide realmente el constructo de la actitud parental ante el conflicto (autoinformada y referenciada) y no otros constructos, y con la validez discriminante, que indica que los dos factores del cuestionario evalúan aspectos diferenciados. En ambos casos, los datos constituyen buenos indicadores de validez (Fornell y Larcker, 1981). Finalmente, la fiabilidad de ambos factores fue satisfactoria, tanto para la estimación del coeficiente de alpha de Cronbach (Bonett y Wright, 2015) como para la fiabilidad compuesta (Carmines y Zeller, 1979; Nunnally, 1978).

Para las profesiones que intervienen con progenitores en conflicto interparental el disponer de un instrumento que mida la actitud hacia el mismo resulta de enorme utilidad. Como señalan Zhou y Buehler (2017), medir la actitud propia y del otro progenitor ante un conflicto posibilita el desarrollo del conflicto constructivo o cooperativo. En este caso, responder al cuestionario requiere que cada progenitor reflexione sobre su deseo de finalizar el conflicto y de llegar a acuerdos, a la vez que sobre el esfuerzo que está dispuesto a realizar para lograrlo; lo que puede ayudar a que sean más conscientes de su posicionamiento, implicación y compromiso en la resolución del conflicto. Igualmente, valorar las ganas que el otro progenitor presenta por alcanzar acuerdos y concluir con el conflicto, a la vez que establecer el nivel de esfuerzo que está dispuesto a llevar a cabo, puede favorecer un proceso empático en ellos. Tener empatía con la percepción del otro progenitor sobre el conflicto es esencial, ya que permite avanzar en las dimensiones del conflicto parental (Zhou y Buehler, 2017).

Además, la información que este cuestionario aporta a los profesionales sobre el deseo de cada progenitor de finalizar el conflicto y llegar a acuerdos, así como el esfuerzo que está dispuesto a realizar, junto con la percepción que tiene del otro sobre estas cuestiones ayuda a entender las posiciones de ambos y a diseñar una mejor estrategia de intervención, para conseguir una resolución constructiva del conflicto. Ya sea en la evaluación de custodias (Fariña, Redondo, Corrás, y Vilariño, 2017; Lund, 2015; Nielsen, 2017), en la mediación familiar (Fariña, 2019) o en la coordinación de parentalidad (D'Abate, 2016; Quigley y Cyr, 2017), terapia de familia (Booth, Crouter, Clements, y Boone-Holladay, 2016; Smyth y Moloney, 2019; Tavassolie et al., 2016), terapia infantil (Zemp et al., 2016) o en la intervención con progenintores en proceso de seperación (Novo, Fariña, Seijo, Vázquez, y Arce, 2019), es factible transformar el conflicto familiar, comprendiendo el de alta intensidad, en una oportunidad de cambio positivo y crecimiento de las personas implicadas (Treloar, 2019). Para lograrlo los profesionales han de disponer del mayor número de herramientas fiables y válidas en las que basar el diagnóstico del caso y la intervención, el cuestionario ACPar se nos presenta como una de ellas.

En cuanto a las limitaciones del presente estudio, es preciso señalar que no se ofrece evidencia acerca de su validez externa, tanto convergente, con instrumentos de medida que evalúen constructos similares, como discriminante, con instrumentos que midan constructos hipotéticamente relacionados con la actitud parental ante el conflicto. Futuras líneas de investigación pueden centrarse en analizar cómo la actitud parental percibida ante un conflicto se relaciona con variables relacionadas como los estilos de crianza (Smetana, 2017), de apego (Jones, Cassidy, y Shaver, 2015) o el funcionamiento familiar en presencia de conflicto (Coe, Davies, y SturgeApple, 2017).

En conclusión, el cuestionario Actitud ante el Conflicto Parental, Autoinformada y Referenciada (ACPar) constituye un instrumento 
válido y fiable para evaluar la percepción de la propia actitud ante un conflicto y de la actitud del otro progenitor, en progenitores hispanohablantes, pudiendo ser de gran valor para la práctica profesional, principalmente, de la mediación, la evaluación de custodia, la coordinación de parentalidad y la terapia familiar.

- Conflicto de intereses.

Los autores declaran no tener ningún conflicto de intereses.

\section{REFERENCIAS}

American Educational Research Association, American Psychological Association, and National Council on Measurement in Education. (2014). The standards for educational and psychological testing. Washington, DC: AERA Publications Sales.

Bonett, D. G., y Wright, T. A. (2015). Cronbach's alpha reliability: Interval estimation, hypothesis testing, and sample size planning. Journal of Organizational Behavior, 36(1), 3-15. https:// doi.org/10.1002/job.1960

Booth, A., Crouter, A. C., Clements, M. L., y Boone-Holladay, T. (2016). Couples in conflict. New York, NY: Routledge.

Camisasca, E., Miragoli, S., y Di Blasio, P. (2016). Families with distinct levels of marital conflict and child adjustment: Which role for maternal and paternal stress? Journal of Child and Family Studies, 25(3), 733-745. https://doi. org/10.1007/s10826-015-0261-0

Camisasca, E., Miragoli, S., y Di Blasio, P. (2019). Children's triangulation during inter-parental conflict: Which role for maternal and paternal parenting stress? Journal of Child and Family Studies, 28(6), 1623-1634. https://doi. org/10.1007/s10826-019-01380-1

Carmines, E. G., y Zeller, R. A. (1979). Reliability and validity assessment. Thousand Oaks, CA: Sage. https://doi.org/10.4135/9781412985642

Coe, J. L., Davies, P. T., y Sturge-Apple, M. L. (2017). The multivariate roles of family instability and interparental conflict in predicting children's representations of insecurity in the family system and early school adjustment problems. Journal of Abnormal Child Psychology, 45(2), 211 224. https://doi.org/10.1007/s10802-0160164-6

Corrás, T., Seijo, D., Fariña, F., Novo, M., Arce, R., y Cabanach, R. G. (2017). What and how much do children lose in academic settings owing to parental separation? Frontiers in Psychology, 8, 1545. https://doi.org/10.3389/ fpsyg.2017.01545

Cummings, E. M., y Davies, P. T. (2002). Effects of marital conflict on children: Recent advances and emerging themes in process-oriented research. Journal of hild Psychology and Psychiatry, 43, 31-63. https://doi.org/10.1111/1469. $\underline{7610.00003}$

Cummings, E. M., y Davies, P. T. (2010). Marital conflict and children: An emotional security perspective. New York, NY: Guilford Press.

Cutrín, O., Maneiro, L., Sobral, J., y GómezFraguela, J. A. (2019). Longitudinal effects of parenting mediated by deviant peers on violent and non-violent antisocial behaviour and substance use in adolescence. European Journal of Psychology Applied to Legal Context, $17(1), \quad 23-32 . \quad$ https://doi.org/10.5093/ eipalc2018a12

D'Abate, D. (2016). Use of solution-focused and family narrative approaches in working with high conflict families: Strategies and techniques that can be utilized in parenting coordination and co-parenting coaching. Journal of Child Custody, 13, 269-288. https://doi.org/10.10 80/15379418.2016.1247308

Dillon, L. M., Nowak, N., Weisfeld, G. E., Weisfeld, C. C., Shattuck, K. S., Imamoğlu, O. E., ... Shen, J. (2015). Sources of marital conflict in five cultures. Evolutionary Psychology, 13(1), 147470491501300101. https://doi. org/10.1177/147470491501300101

Fariña, F. (2019). La mediación familiar, una manifestación de Justicia Terapéutica [Family mediation, a manifestation of Therapeutic Jurisprudence]. En I. Luján-Henríquez (Ed.), Conflictos y mediación en contextos plurales de convivencia (pp. 121-144). Las Palmas de Gran Canaria, España: Servicio de Publicaciones y Difusión Científica de la ULPGC.

Fariña, F., Redondo, L., Corrás, T., y Vilariño, M. 
(2017). Study of the effects of anchorage in judicial judgements in child custody dispute proceedings. Acción Psicológica, 14(2), 147 155. https://doi.org/10.5944/ap.14.2.21239 Fornell, C., y Larcker, D. F. (1981). Evaluating structural equation models with unobservable variables and measurement error. Journal of Marketing Research, 18(1), 39-50. https://doi. org/10.1177/002224378101800104

Fosco, G. M., y Feinberg, M. E. (2014). Cascading effects of interparental conflict in adolescence: Linking threat appraisals, selfefficacy, and adjustment. Development and Psychopathology, 27(1), 239-252. https://doi. org/10.1017/s0954579414000704

Frankel, L. A., Umemura, T., Jacobvitz, D., y Hazen, N. (2015). Marital conflict and parental responses to infant negative emotions: Relations with toddler emotional regulation. Infant Behavior and Development, 40, 73-83. https:// doi.org/10.1016/i.infbeh.2015.03.004

Gallego, R., Novo, M., Fariña, F., y Arce, R. (2019). Child-to-parent violence and parent-to-child violence: A meta-analytic review. European Journal of Psychology Applied to Legal Context, $17(2), \quad 51-59 . \quad$ https://doi.org/10.5093/ ejpalc2019a4

Gao, M. M., Du, H., Davies, P. T., y Cummings, E. M. (2018). Marital conflict behaviors and parenting: Dyadic links over time. Family Relations, 68(1), 135-149. https://doi. org/10.1111/fare.12322

Gómez-Ortiz, O., Romera, E. M., JiménezCastillejo, R., Ortega-Ruiz, R., y García-López, L. J. (2019). Parenting practices and adolescent social anxiety: A direct or indirect relationship? International Journal of Clinical and Health Psychology, 19(2), 124-133. https://doi. org/10.1016/i.ijchp.2019.04.001

Gorsuch, R. L. (1997). Exploratory factor analysis: Its role in item analysis. Journal of Personality Assessment, 68, 532-560. https://doi. org/10.1207/s15327752ipa6803 5

Grych, J. H., Seid, M., y Fincham, F. D. (1992). Assessing marital conflict from the child's perspective: The Children's Perception of Interparental Conflict Scale. Child Development, 63(3), 558-572. https://doi. org/10.1111/i.1467-8624.1992.tb01646.x

Iraurgi, I., Martínez-Pampliega, A., Sanz, M., Cosgaya, L., Galíndez, E., y Muñoz, A. (2008). Escala de Conflicto Interparental desde la Perspectiva de los Hijos (CPIC): Estudio de validación de una versión abreviada de 36 ítems [Children's Perception of Interparental Conflict Scales (CPIC): Validation study of a short version of 36 items]. Revista Iberoamericana de Diagnóstico y Evaluación-e Avaliação Psicológica, 7 (25), 9-34. Recuperado de http:// www.aidep.org/03 ridep/R25/R251.pdf

Jones, J. D., Cassidy, J., y Shaver, P. R. (2015). Parents' self-reported attachment styles: A review of links with parenting behaviors, emotions, and cognitions. Personality and Social Psychology Review, 19(1), 44-76. https://doi. org/10.1177/1088868314541858

Kerig, P. K. (1996). Assessing the links between interparental conflict and child adjustment: The conflicts and problem-solving scales. Journal of Family Psychology, 10(4), 454. https://doi. org/10.1037//0893-3200.10.4.454

Khurshid, S., Peng, Y., y Wang, Z. (2019). Respiratory sinus arrhythmia acts as a moderator of the relationship between parental marital conflict and adolescents' internalizing problems. Frontiers in Neuroscience. 13, 500. https://doi.org/10.3389/fnins.2019.00500

Kimmel, D., y Van Der Veen, F. (1974). Factors of marital adjustment in Locke's Marital Adjustment Test. Journal of Marriage and the Family, 36(1), 57-63. https://doi.org/10.2307/350994

Kjøs, P., y Weie-Oddli, H. (2018). Alliance formation in high conflict custody mediation: A serial case analysis. Journal of Family Therapy, 40(3), 378-397. https://doi.org/10.1111/14676427.12180

Kline, R. B. (2011). Principles and practice of structural equation modeling (3a. ed.). New York, NY: Guilford.

Lamela, D., Figueiredo, B., Bastos, A., y Feinberg, M. (2015). Typologies of post-divorce coparenting and parental well-being, parenting quality and children's psychological adjustment. Child Psychiatry \& Human Development, 47(5), 716-728. https://doi.org/10.1007/s10578$\underline{015-0604-5}$ 
Laursen, B. (1993). The Perceived Impact of Conflict on Adolescent Relationships. MerrillPalmer Quaterly, 38(4), 535-550. Retrieved from http://www.jstor.org/stable/23087248

Lucas-Thompson, R. G., Lunkenheimer, E. S., y Dumitrache, A. (2017). Associations between marital conflict and adolescent conflict appraisals, stress physiology, and mental health. Journal of Clinical Child \& Adolescent Psychology, 46(3), 379-393. https://doi.org/l $\underline{0.1080 / 15374416.2015 .1046179}$

Lund, M. E. (2015). The place for custody evaluations in family peacemaking. Family Court Review, 53(3), 407-417. https://doi. org/10.1111/fcre.12162

Martínez-Pampliega, A., Ugarte, I., Merino, L., y Herrero-Fernández, D. (2019). Conciliación familia-trabajo y sintomatología externalizante de los hijos e hijas: Papel mediador del clima familiar [Work-family balance and children's external simptomatology: the mediating role of family climate]. Revista Iberoamericana de Psicología y Salud., 10(1), 27-36. https://doi. org/10.23923/i.rips.2018.02.023

Martinón, J. M., Fariña, F., Corrás, T., Seijo, D., Souto, A., y Novo, M. (2017). Impacto de la ruptura de los progenitores en el estado de salud física de los hijos [Impact of parental breakup on the physical health of children]. European Journal of Education and Psychology, 10, 9-14. https://doi.org/10.1016/i.ejeps.2016.10.002

Moos, R. H., Moos, B. S., y Trickett, E. J. (1984): Escalas de Clima Social [Social Climate Scales]. Madrid, España: TEA.

Motrico, E., Fuentes, M. J., y Bersabé, R. (2001). Discrepancias en la percepción de los conflictos entre padres e hijos/as a lo largo de la adolescencia. Anales de Psicología, 17(1), $1-13$.

Muñiz, J., y Fonseca-Pedrero, E. (2019). Diez pasos para la construcción de un test [Ten steps for test development]. Psicothema, 31 (1), 7-16. https:// doi.org/10.7334/psicothema2018.291

Myers, N. D., Ahn, S., y Jin, Y. (2011). Sample size and power estimates for a confirmatory factor analytic model in exercise and sport: A Monte Carlo approach. Research Quarterly for Exercise and Sport, 82, 412-423. https://doi.or $\mathrm{g} / 10.1080 / 02701367.2011 .10599773$

Nielsen, L. (2017). Re-examining the research on parental conflict, coparenting, and custody arrangements. Psychology, Public Policy, and Law, 23(2), 211 -231. https://doi.org/10.1037/ law0000109

Novo, M., Fariña, F., Seijo, D., Vázquez, M. J., y Arce, R. (2019). Assessing the effects of a parental separation education program on mental health problems. Psicothema, 31 (3), 284-291. https://doi.org/10.7334/ psicothema2018.299

Nunnally, J.C. (1978). Psychometric theory. New York, NY: McGraw-Hill.

Parra-Jiménez, Á. P., y Oliva-Delgado, A. O. (2002). Comunicación y conflicto familiar durante la adolescencia [Family communication and conflict during adolescence] Anales de Psicología, 18(2), 215-231. Recuperado de https://revistas.um.es/analesps/article/ view/28421

Pituch, K. A., y Stevens, J. P. (2016). Applied multivariate statistics for the social sciences. Ney York, NY: Routledge.

Quigley, C., y Cyr, F. (2017) Children's perspectives on parenting coordination: Insights from the Montreal Parenting Coordination Pilot Project. Journal of Child Custody, 14(2-3), 151-174. https://doi.org/0.1080/15379418.2017.137 1093

Schermelleh-Engel, K., Moosbrugger, H., y Müller, $\mathrm{H}$. (2003). Evaluating the fit of structural equation models: Tests of significance and descriptive goodness-of-fit measures. Methods of Psychological Research Online, 8(2), 23-74.

Seijo, D., Fariña, F., Corras, T., Novo, M., y Arce, R. (2016). Estimating the epidemiology and quantifying the damages of parental separation in children and adolescents. Frontiers in Psychology, 7, 1611. https://doi.org/10.3389/ fpsyg.2016.01611

Shamir, H., Schudlich, T. D. R., y Cummings, E. M. (2001). Marital conflict, parenting styles, and children's representations of family relationships. Parenting: Science and Practice, 1, 123-151 https://doi.org/10.1080/152951 92.2001 .9681214

Smetana, J. G. (2017). Current research on 
parenting styles, dimensions, and beliefs. Current Opinion in Psychology, 15, 19-25. https://doi. org/10.1016/i.copsyc.2017.02.012

Smyth, B. M., y Moloney, L. J. (2019). Postseparation parenting disputes and the many faces of high conflict: Theory and research. Australian and New Zealand Journal of Family Therapy, 40,74-84. https://doi.org/10.1002/ anzf. 1346

Straus, M. A., Lamby, S. L., Boney-McCoy, S., y Savage, S. A. (1995). The Revised Conflict Tactics Scale (CTS2). Development and preliminary psychometric data. Journal of Family Issues, 17, 283-316. https://doi. org/10.1177/019251396017003001

Tabanick, B. G., y Fidell, L. S. (1989). Using multivariate statistics. New York, NY: Harper Collins.

Tavassolie, T., Dudding, S., Madigan, A. L., Thorvardarson, E., y Winsler, A. (2016). Differences in perceived parenting style between mothers and fathers: Implications for child outcomes and marital conflict. Journal of Child and Family Studies, 25(6), 2055-2068. doi 10.1007/s10826-016-0376-y

Treloar, R. (2019). Parents making meaning of high conflict divorce. Australian and New Zealand Journal of Family Therapy, 40, 85-97. https:// doi.org/10.1002/anzf. 1347

Tu, K. M., Erath, S. A., y El-Sheikh, M. (2016). Coping responses moderate prospective associations between marital conflict and youth adjustment. Journal of Family Psychology, 30(5), 523-532. https://doi.org/10.1037/ fam0000169

Van der Wal, R. C., Finkenauer, C., y Visser, M. M. (2019). Reconciling mixed findings on children's adjustment following high-conflict divorce. Journal of Child and Family Studies, 28(2), 468-478. https://doi.org/10.1007/ s10826-018-1277-z

Yap, M. B. H., y Jorm, A. F. (2015). Parental factors associated with childhood anxiety, depression, and internalizing problems: A systematic review and meta-analysis. Journal of Affective Disorders, 175, 424-440. https://doi. org/10.1016/i.jad.2015.01.050

Yárnoz-Yaben, S., y Comino-González, P. (2010). El CAD-S, un instrumento para la evaluación de la adaptación al divorcio-separación [The CAD-S, an Instrument for the assessment of adaptation to divorce-separation. Psicothema, 22(1), 157-162. Recuperado de http://www. psicothema.com/pdf/3711.pdf

Zemp, M., Bodenmann, G., y Cummings, E. M. (2016). The significance of interparental conflict for children. European Psychologist, 21 (2), 99108. https://doi.org/10.1027/1016-9040/ a000245

Zhou, N., y Buehler, C. (2017). Adolescents' responses to marital conflict: The role of cooperative marital conflict. Journal of Family Psychology, 37(7), 910-921. https://doi. org/10.1037/fam0000341 


\section{ANEXO 1}

\section{•ÍTEMS ORIGINALES}

1 - En general, el nivel de conflicto que existe en su familia es

2- En general, el nivel de conflicto que existe con el otro progenitor es

3- Cuando existe conflicto, su deseo de finalizar con el conflicto

4- Cuando existe conflicto, su deseo de llegar a acuerdos

5- Cuando existe conflicto, el esfuerzo que está dispuesto a realizar para finalizar el conflicto

6- Cuando existe conflicto, el esfuerzo que está dispuesto a realizar para llegar a acuerdos

7- Cuando existe conflicto, el deseo del otro progenitor de finalizar con el conflicto

8- Cuando existe conflicto, el deseo del otro progenitor de llegar a acuerdos

9- Cuando existe conflicto, el esfuerzo que está dispuesto a realizar el otro progenitor para finalizar el conflicto

10- Cuando existe conflicto, el esfuerzo que está dispuesto a realizar el otro progenitor para llegar a acuerdos

11 - Cuando existe conflicto, la responsabilidad que tiene usted en el conflicto

12- Cuando existe conflicto, la responsabilidad que tiene el otro progenitor en el conflicto

13- Cuando existe conflicto, la responsabilidad que tienen otras personas en el conflicto (indique quienes...)

14 - Cuando existe conflicto, el llegar a acuerdos depende de usted

15- Cuando existe conflicto, el llegar a acuerdos depende del otro progenitor

16- Cuando existe conflicto, el llegar a acuerdos depende de otras personas

17- Cuando existe conflicto, lo que le importa a usted el bienestar de sus hijos/as

\section{ANEXO 2}

\section{-VERSIÓN DEFINITIVA DEL INSTRUMENTO}

Considerando que las controversias y los conflictos son inherentes a las relaciones humanas, y también entre progenitores, valore la intensidad de los siguientes aspectos en relación con el otro progenitor, en una escala del 0 (nada o ninguno) y 10 (máximo o total).

AA 1 - Cuando existe conflicto, su deseo de finalizar con el conflicto

AA2- Cuando existe conflicto, su deseo de llegar a acuerdos

AA3- Cuando existe conflicto, el esfuerzo que está dispuesto a realizar para finalizar el conflicto

AA4- Cuando existe conflicto, el esfuerzo que está dispuesto a realizar para llegar a acuerdos

AR 1 - Cuando existe conflicto, el deseo del otro progenitor de finalizar con el conflicto

AR2- Cuando existe conflicto, el deseo del otro progenitor de llegar a acuerdos

AR3- Cuando existe conflicto, el esfuerzo que está dispuesto a realizar el otro progenitor para finalizar el conflicto

AR4- Cuando existe conflicto, el esfuerzo que está dispuesto a realizar el otro progenitor para llegar a acuerdos 